\title{
Infarto de miocardio ST elevado después de un trauma eléctrico: Una rara complicación
}

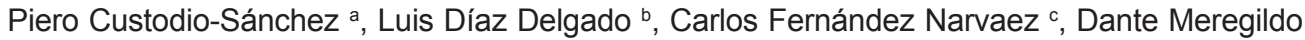 \\ Rodríguez c , Milagros Ochoa Medina a , Ciro Dávila Díaz a, Victor Castro Ramos a,, \\ Hernán Vela Jara ${ }^{a}$, ,Vanessa Castillo Atoche ${ }^{a}$,
}

\section{RESUMEN}

Las descargas eléctricas pueden provocar una amplia gama de afecciones clínicas, desde quemaduras en la piel hasta arritmias fatales. La incidencia de efectos cardiacos es variable, siendo las principales complicaciones las arritmias y las injurias del tejido miocárdico. Las arritmias más frecuentes son la taquicardia sinusal y las extrasístoles ventriculares. El infarto agudo de miocardio ST elevado es una complicación rara que puede conllevar resultados fatales; en la mayoría de casos reportados se han encontrado arterias coronarias normales y se considera el vasoespasmo coronario como la etiología. Presentamos un caso atendido en la emergencia de un hospital del norte de Perú.

Palabras clave: Traumatismo por electricidad, Infarto agudo de miocardio, Vasoespasmo coronario.(Fuente: DeCS-BIREME)

\section{ST Elevation Myocardial Infarction after an electric shock: A rare complication}

\section{ABSTRACT}

Electric shocks can cause a wide range of clinical conditions from skin burns to fatal arrhythmias. The incidence of cardiac effects is variable, the main complications are arrhythmias and myocardial injuries. The most frequent arrhythmias are sinus tachycardia and ventricular ectopic beats. Acute myocardial infarction with ST-segment elevation is a rare complication that can lead to fatal results. Normal coronary arteries have been reported in most of the cases and vasospasm is thought to be the etiology. We present a case attended in the emergency of a hospital in northern Peru.

Keywords: Electric shock, Acute myocardial infarction, Coronary vasospasm.(Source: MeSH-NLM)

a Médico Cardiólogo. Hospital Regional Lambayeque. Chiclayo. Perú.

${ }^{\mathrm{b}}$ Residente de Medicina Interna. Hospital Regional Lambayeque. Chiclayo. Perú.

${ }^{c}$ Médico Internista. Hospital Regional Lambayeque. Chiclayo. Perú. 
INTRODUCCIÓN

En Estados Unidos anualmente 4400 personas aproximadamente son víctimas de traumatismos por electricidad y 400 personas mueren debido a este motivo ${ }^{(1,2)}$. Las complicaciones cardiacas generalmente son de presentación variable (14 - 54\%), siendo las arritmias y las injurias del tejido miocárdico las principales.

Las arritmias son las complicaciones de mayor frecuencia, siendo las más frecuentes la taquicardia sinusal, las extrasístoles ventriculares, la fibrilación/flutter auricular, la taquicardia ventricular o la fibrilación ventricular (2).

La injuria miocárdica, determinada por una elevación de las troponinas cardiacas (3), puede producirse por diferentes mecanismos como: Injuria directa, injuria mediada por liberación de catecolaminas a la microcirculación, reducción del flujo coronario secundario a hipotensión generalizada, espasmo/trombosis coronaria, o contusión miocárdica relacionada a la reanimación cardiopulmonar (4). La presencia de injuria miocárdica con evidencia clínica de isquemia miocárdica aguda, define el infarto agudo de miocardio.

La presencia de un infarto de miocardio ST elevado (IM STE) después de un trauma eléctrico, es raramente visto y su patogénesis es controversial, siendo considerado el vasoespasmo coronario, el mecanismo etiológico principal. Tras el vasoespasmo coronario, puede ocurrir un desequilibrio miocárdico entre el aporte y la demanda de oxígeno, no relacionado con una aterotrombosis aguda, lo que se conoce como infarto agudo de miocardio tipo 2 (3), el cual puede presentarse con síntomas clínicos clásicos y patrones electrocadiográficos similares al infarto de miocardio aterotrombótico, incluyendo la elevación del segmento ST o

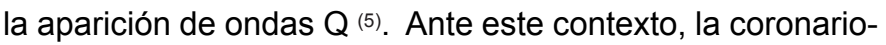
grafía debe ser realizada si hay duda sobre la posibilidad de una enfermedad coronaria subyacente, ya que una coronariografia normal ha sido reportada en la mayoría de casos ${ }^{(6)}$.

En reportes previos, Celebi y col. (7) registraron un IM STE inferior, con coronarias normales, manejado con betabloqueantes e inhibidores de enzima convertidora de angiotensina (IECA); Belen y col.(6) registraron un IM STE inferior, con coronarias normales, manejado con ácido acetilsalicílico, betabloqueantes e IECA; Behcet y col. (8) registraron un IM STE inferior, con coronarias normales, manejado con ácido acetilsalicílico, betabloqueantes e IECA; Gursul y col. (6) registraron un IM STE inferior, con coronarias normales; y Jacob y col. (9) registraron un infarto de miocardio evolutivo, con trombosis y disección multivaso, revascularizado mediante cirugía de bypass.

Otras complicaciones cardiovasculares descritas incluyen cambios inespecíficos del segmento ST, prolongación del QT, disturbios de conducción como bradicardia sinusal, pa - rada cardiaca, bloqueos de rama, bloqueos auriculoventriculares, pericarditis hemorrágica, hipertensión arterial transitoria, disfunción autonómica transitoria e injurias vasculares (10).

En Perú, la unidad de quemados del Hospital Nacional Guillermo Almenara Irigoyen informa que sólo el 9\% presentó alguna complicación cardiológica, sin registrarse algún caso de infarto agudo de miocardio (11); y en un informe reciente de Corea, las complicaciones cardiacas ocurrieron en un $14.8 \%$ de pacientes, de los cuales $72.9 \%$ presentó elevación de troponina I, 3.7\% anormalidades de motilidad regional y $5 \%$ flutter/fibrilación auricular, siendo este grupo de mayor mortalidad que el grupo control, y el área de superficie corporal total el único factor de riesgo asociado (12).

El objetivo del presente caso es reportar el diagnóstico y manejo desafiante del infarto de miocardio ST elevado después de un traumatismo por electricidad, a propósito de un caso acontecido en un hospital del norte del Perú

\section{REPORTE DE CASO}

Varón de 52 años, sin antecedentes de importancia, obrero de construcción. Sufre contacto accidental con cables de alta tensión sufriendo descarga eléctrica, con pérdida de conciencia por aproximadamente 15 minutos, presentando al despertar dolor torácico retroesternal opresivo de moderada intensidad, sin asociarse a síntomas neurovegetativos. Es llevado a emergencia del hospital aproximadamente con 30 minutos de evolución, presentando al ingreso una PA:100/60 mmHg, FC 82 Ipm, FR 16 rpm: SpO2: $96 \%$, fiO2: 0.21, y al examen físico, quemaduras de segundo grado superficiales y profundas en manos, tronco, pierna izquierda y pie derecho (47\% de área de superficie corporal) (Figura 3). El electrocardiograma inicial, tomado a los 10 minutos de su ingreso, mostró elevación de $5 \mathrm{~mm}$ del segmento ST en derivadas II, III, AVF, e infradesnivel del ST en derivadas aVL, V1 y V2 (Figura 1). Tras su ingreso cursó con disminución progresiva y espontánea del dolor torácico hasta su completa desaparición tras 30 minutos.

El ecocardiograma inicial, a la hora de su ingreso, mostró hipocinesia inferolateral y lateroapical, con función sistólica del ventrículo izquierdo conservada. Se diagnosticó síndrome coronario agudo ST elevado inferoposterior, secundario a un probable vasoespasmo coronario. Luego se continuó con el tratamiento médico con ácido acetilsalicílico $100 \mathrm{mg}$ cada 24 horas, atorvastatina $40 \mathrm{mg}$ cada 24 horas y bisoprolol $5 \mathrm{mg}$ cada 24 horas. En el electrocardiograma control a las 12 horas se apreció nuevas ondas $Q$ patológicas, como signos de necrosis en cara inferior 
(Figura 2). Las troponinas tomadas a la hora de su ingreso presentaron un ascenso posterior, y se evidenció además aumento de transaminasas y elevación de creatinfosfoquinasa (Tabla 1). El paciente ingresó al área de observación para monitoreo continuo, curación de heridas y manejo multidisciplinario. Durante su hospitalización permaneció asintomático, hemodinámicamente estable, sin presentar recurrencia del dolor o complicaciones cardiovasculares; cursó con evolución favorable de lesiones dérmicas, siendo dado de alta a las tres semanas, para seguimiento por consultorio externo y referencia para coronariografía electiva.

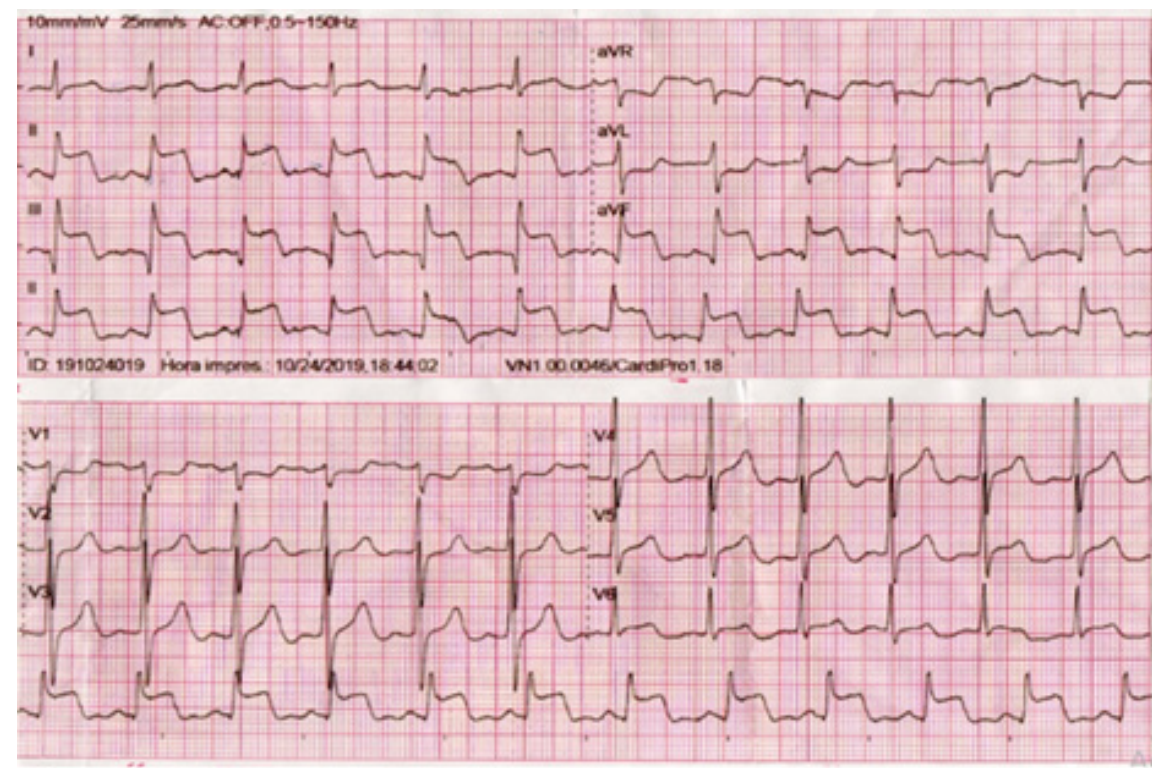

Figura 1. Electrocardiograma al ingreso, con supradesnivel del ST inferior.

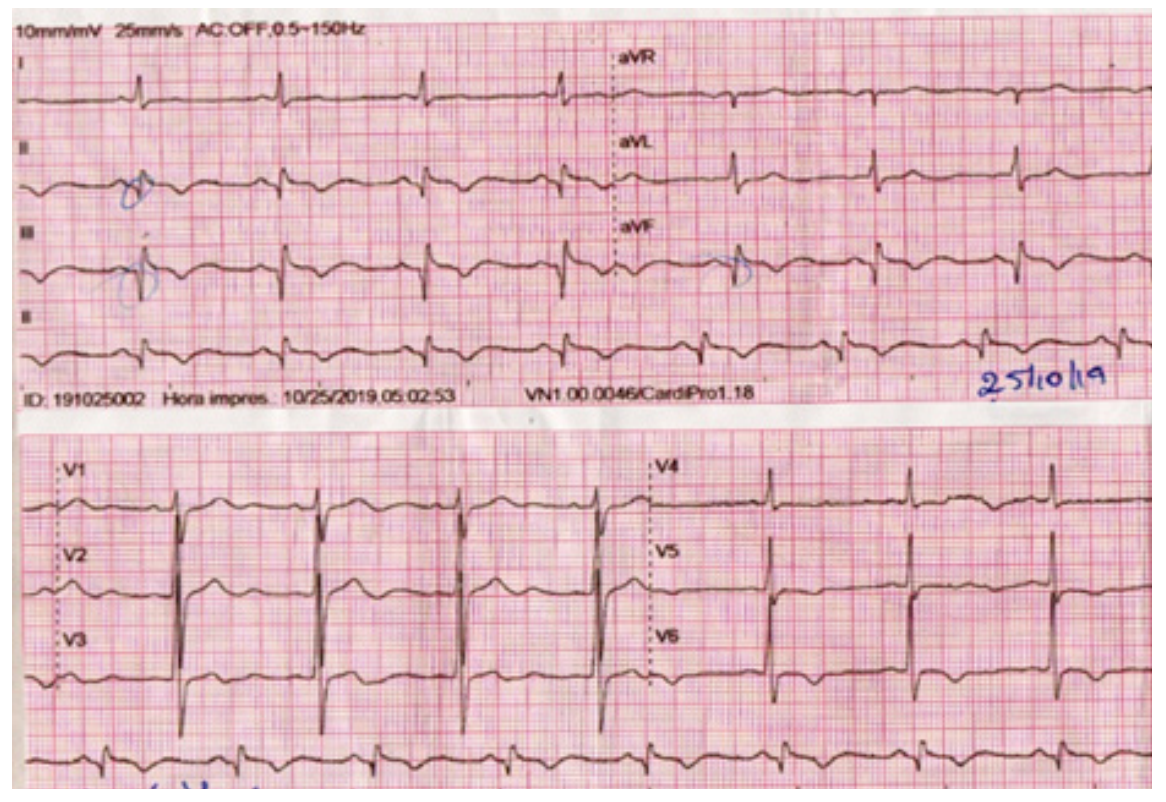

Figura 2. Electrocardiograma a las 12 horas, con signos de necrosis e isquemia inferior.. 


\begin{tabular}{lllll}
\hline \multirow{2}{*}{$\begin{array}{l}\text { Tipo de } \\
\text { análisis }\end{array}$} & $\begin{array}{l}\text { Valores } \\
\text { normales }\end{array}$ & \multicolumn{3}{c}{ Días de hospitalización } \\
\cline { 3 - 5 } & & \multicolumn{2}{c}{0} & 1 \\
\hline AST & $15-37 \mathrm{U} / \mathrm{L}$ & 1057 & 1280 & \\
ALT & $30-65 \mathrm{U} / \mathrm{L}$ & 678 & 890 & \\
LDH & $105-333$ & & 600 & \\
& $\mathrm{U} / \mathrm{L}$ & & & \\
PCR & $<10 \mathrm{mg} / \mathrm{L}$ & & 27 & \\
Fibrinógeno & $200 \mathrm{a} 400$ & & 560 & \\
& $\mathrm{mg} / \mathrm{dl}$ & & & \\
Troponina T & $<0.01 \mathrm{ng} / \mathrm{ml}$ & 0.013 & 0.5 & \\
Troponina I & $<0.01 \mathrm{ng} / \mathrm{ml}$ & 0.08 & 1.1 & \\
CPK-MB & $<5 \mathrm{nd} / \mathrm{dl}$ & & 190 & 219 \\
CPK Total & $<200 \mathrm{U} / \mathrm{L}$ & & 413 & 24580 \\
\hline \hline
\end{tabular}

Tabla 1. Exámenes de laboratorio al ingreso.

ALT: Alanina aminotransferasa, AST: Aspartato aminotransferasa, LDH: lactato deshidrogenasa, PCR: Proteína $C$ reactiva, CPK - MB: Creatinfosfoquinasa, fracción MB, CPK: Creatinfosfoquinasa.

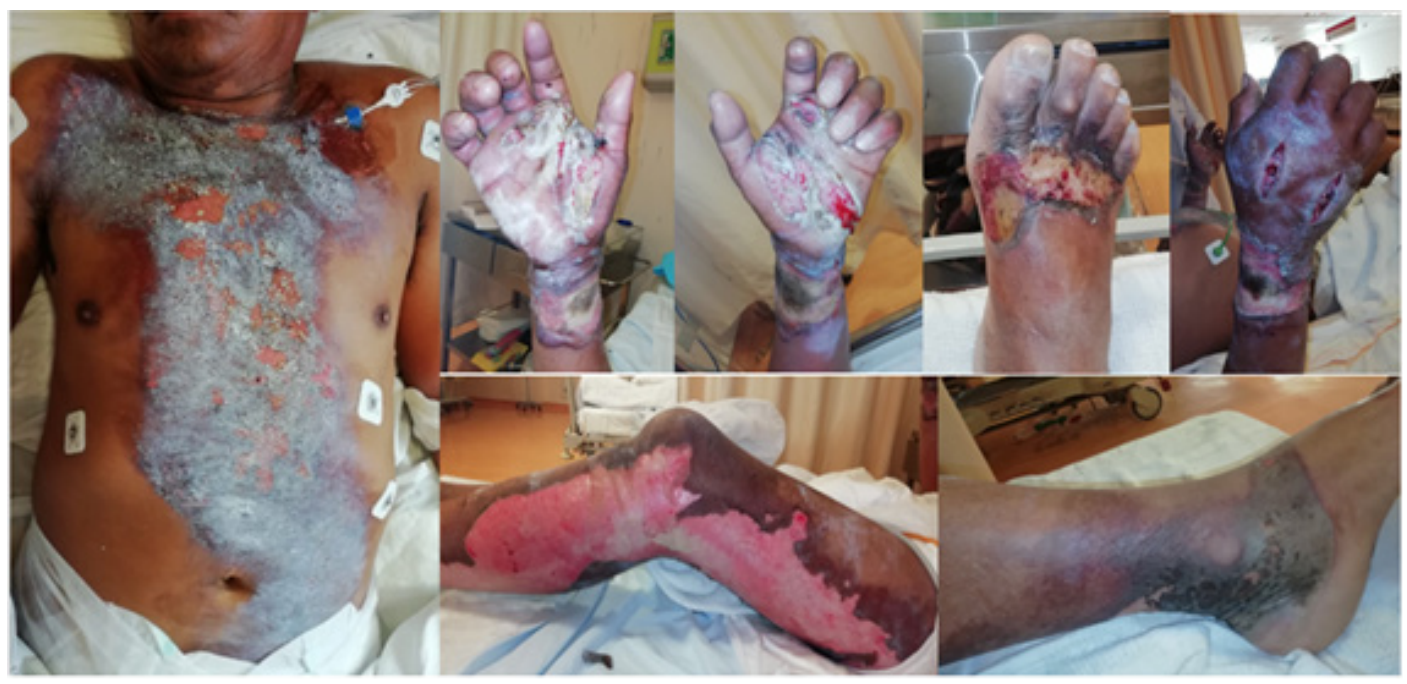

Figura 3. Quemaduras de segundo grado superficiales y profundas.

\section{DISCUSIÓN}

El infarto de miocardio es una potencial complicación después de un trauma eléctrico que es raramente visto y su patogénesis permanece controversial ${ }^{(1-3)}$. En nuestro paciente, el diagnóstico de infarto de miocardio ST elevado inferoposterior fue concluyente por la clínica, así como los resultados del electrocardiograma, el ecocardiograma y las enzimas cardíacas; pero al no contarse con sala de hemodinámica disponible, considerando el traumatismo severo del paciente (contraindicación absoluta para fibrinólisis), la resolución del dolor torácico, y sospechando de vasoespasmo coronario como el mecanismo fisiopatológico subyacente que originó un desequilibrio de oxígeno e isquemia aguda (infarto de miocardio tipo 2), no se consideró el tratamiento fibrinolítico urgente como estrategia inicial. No hay consenso del manejo del infarto agudo de miocardio ST elevado después de un trauma eléctrico; y no existe datos que sugieran beneficio de antiplaquetarios o terapia anticoagulante para aquellos con un infarto tipo 2, coronario no aterotrombótico, por lo que el tratamiento debe ser individualizado ajustado a las características específicas de cada paciente ${ }^{(13)}$. 
La coronariografía es de elección para evaluar el mecanismo etiológico subyacente y contribuye a guiar la terapia, ya que se han reportado en la mayoría de casos arterias coronarias normales o sin lesiones obstructivas, siendo el vasoespasmo coronario el mecanismo principal ${ }^{(5,6)}$. Así, la angioplastia percutánea estaría indicada solo en aquellos casos en que se encuentren lesiones coronarias oclusivas como mecanismo responsable ${ }^{(4,7,8)}$.

En reportes previos un electrocardiograma anormal fue encontrado en aproximadamente $31 \%$ de los pacientes tras una descarga eléctrica. Dada la naturaleza no transmural de la necrosis, la elevación del segmento ST ocurre raramente, siendo el supradesnivel en derivadas inferiores, como nuestro caso, lo más frecuentemente observado. Este predominio es explicado por la proximidad de la coronaria derecha a la superficie corporal que la hace más vulnerable ${ }^{(6,8)}$. Los cambios electrocardiográficos pueden normalizarse y tienden a ser totalmente reversibles en los supervivientes a largo plazo. La CPK - MB es menos específica, ya que puede incrementarse marcadamente con la injuria muscular esquelética concomitante, por lo que la troponina es más útil y debería ser preferida en estos pacientes. La ecocardiografía, puede revelar hipoquinesia difusa o regional y estos hallazgos pueden mejorar marcadamente en el seguimiento ${ }^{(5,8,14)}$.

Se concluye que el infarto de miocardio ST elevado es una rara complicación reportada después de un trauma eléctrico, generalmente relacionada a vasoespasmo coronario (infarto de miocardio tipo 2), por lo que, en ausencia de una coronariografía de urgencia, un manejo conservador es una alternativa adecuada con buen resultado.

Es recomendable que pacientes, que presenten injurias eléctricas de alto voltaje, síncope o una disminución del nivel de conciencia, dolor de pecho, cualquier anormalidad del ritmo o evidencia de isquemia, deberían tener un electrocardiograma y monitoreo cardiaco continuo en su evaluación inicial ${ }^{(10)}$.

\section{Fuente de financiamiento: Ninguna}

\section{Conflicto de interéses: Ninguna}

\section{REFERENCIAS BIBLIOGRAFICAS}

1. Waldmann V, Narayanan K, Combes N, Jost D, Jouven X, Marijon E. Electrical cardiac injuries: current concepts and management. Eur Heart J. 2018; 39(16):1459-1465.

2. Celebi A, Gulel O, Cicekcioglu H, Gokaslan S, Kututcularoglu G, Ulusoy V. Myocardial infarction after an electric shock: a rare complication. Cardiol J. 2009;16(4):362-364.

3. Thygesen K, Alpert J, Jaffe A, Chaitman B, Bax J, Morrow D, White H. Consenso ESC 2018 sobre la cuarta definición universal del infarto de miocardio. Rev Esp Cardiol. 2019; 72(1): 72.e1-e27.

4. Gursul E, Bayata S, Aksit E, Ugurlu B. Development of ST Elevation Myocardial Infarction and Atrial Fibrillation after an Electrical Injury. Case Rep Emerg Med. 2015; 2015:953102.

5. Ertem AG, Efe TH, Dogan M, Yeter E, Sevim Y. A rare cause of acute coronary syndrome: electrical shock. Wien Klin Wochenschr. 2013;125(1516): 487-489.

6. Belen E, Tipi FF, Bayyiğit A, Helvacı AŞ. Acute inferior myocardial infarction after electrical weapon exposure: case report and review of the literature. Turk Kardiyol Dern Ars. 2015 Mar;43(2):178-81.

7. Celebi A, Gulel O, Cicekcioglu H, Gokaslan S, Kututcularoglu G, Ulusoy $\mathrm{V}$. Myocardial infarction after an electric shock: A rare complication. Cardiol J 2009; 16, 4: 362-364

8. Behçet Al, Pınar Yarbil, Hasan Orhan Özer, Selve Aslan, Cuma Yıldırım, Vedat Davutoğlu. A Rare Complication of Electric Shock: Myocardial Infarction. AKATOS 2012; 3(1): 4-8. JAEMCR 2012; 3(1):4-8

9. Jacob S, Belli E. Electric Shock-Induced Coronary Artery Thrombosis and Dissection. Ann Thorac Surg 2019;107(2): e105-106.

10. Gentges J, Schieche C. Electrical injuries in the emergency department: an evidence-based review. Emerg Med Pract. 2018; 20(11):120.

11. Winston La Torre Tang. (2003). Quemaduras eléctricas: Estudio clínico - epidemiológico en el Hospital Guillermo Almenara Irigoyen, 1997 - 2001. Universidad Nacional Mayor De San Marcos, Perú.

12. Choi JH, Han D, Kang SH, Yoon CH, Cho JR, Kym D. Retrospective study of prognosis and relating factors of cardiac complications associated with electrical injuries at a single centre in Korea. BMJ Open 2019; 9(7): e028741.

13. Sandoval Y, Jaffe A. Infarto de miocardio de tipo 2. J Am Coll Cardiol 2019; 73:1846-1860

14. Hansen SM, Riahi S, Hjortshøj S, Mortensen R, Køber L, Søgaard P Torp-Pedersen C. Mortality and risk of cardiac complications among immediate survivors of accidental electric shock: a Danish nationwide cohort study. BMJ Open 2017;7(8): e015967. 